\title{
Immunotherapy of brain metastases: breaking a "dogma"
}

\author{
Anna Maria Di Giacomo ${ }^{1}$, Monica Valente', Alfonso Cerase², Maria Fortunata Lofiego ${ }^{1}$, Francesca Piazzini', \\ Luana Calabrò ${ }^{1}$, Elisabetta Gambale ${ }^{1}$, Alessia Covre ${ }^{1}$ and Michele Maio ${ }^{1 *}$ (iD
}

\begin{abstract}
Until very few years ago, the oncology community dogmatically excluded any clinical potential for immunotherapy in controlling brain metastases. Therefore, despite the significant therapeutic efficacy of monoclonal antibodies to immune check-point(s) across a wide range of tumor types, patients with brain disease were invariably excluded from clinical trials with these agents. Recent insights on the immune landscape of the central nervous system, as well as of the brain tumor microenvironment, are shedding light on the immune-biology of brain metastases. Interestingly, retrospective analyses, case series, and initial prospective clinical trials have recently investigated the role of different immune check-point inhibitors in brain metastases, reporting a significant clinical activity also in this subset of patients. These findings, and their swift translation in the daily practice, are driving fundamental changes in the clinical management of patients with brain metastases, and raise important neuroradiologic challenges. Along this line, neuro-oncology undoubtedly represents an additional area of active investigation and of growing interest to support medical oncologists in the evaluation of clinical responses of brain metastases to $\mathrm{ICI}$ treatment, and in the management of neurologic immune-related adverse events.

Aim of this review is to summarize the most recent findings on brain metastases immunobiology, on the evolving scenario of clinical efficacy of $\mathrm{ICl}$ therapy in patients with brain metastases, as well as on the increasing relevance of neuroradiology in this therapeutic setting.
\end{abstract}

Keywords: Brain metastases, Immune checkpoint(s), Cancer immunotherapy, Lung cancer, Magnetic resonance imaging, Melanoma, Neuroradiology, Tumor microenvironment

\section{Background}

The occurrence of brain metastases in solid tumors is steadily increasing [1]. About $50 \%$ of cancer patients will experience metastatic spreading to the central nervous system (CNS) in the course of their disease [2-4], with the highest incidence been reported in melanoma (28.2\%), lung (26.8\%), renal (10.8\%), and breast cancer (7.6\%) [5]. The prognosis and survival of patients with brain metastases remains poor; relevant prognostic factors include age, primary disease control, presence of extracranial metastases or leptomeningeal disease, and performance status, though their clinical value is limited [6]. The overall 2 and 5 year survival estimates for

\footnotetext{
* Correspondence: mmaiocro@gmail.com

${ }^{1}$ Department of Oncology, Center for Immuno-Oncology, Medical Oncology and Immunotherapy, University Hospital of Siena, Viale Bracci, 14, 53100 Siena, Italy

Full list of author information is available at the end of the article
}

patients who develop brain metastases across different tumor types are 8.1 and $2.4 \%$, respectively, and disease spreading to the CNS represents the cause of death in more than half of these subjects [6]. Therapeutic options for patients with brain metastases are largely palliative and include surgical resection, whole-brain radiation therapy (WBRT), stereotactic radiosurgery (SRS), or their combinations [4], while chemotherapy is uncommonly utilized due to its acknowledged limitation to effectively cross the blood-brain barrier [1]. This latter notion, and the poorer prognosis of patients with brain metastases has led them to be generally excluded from clinical trials with chemotherapeutic agents in the past; a similar scenario applied also more recently to immunotherapy with immune check-point inhibitors (ICI) [7]. However, in the last years, many scientific efforts were directed to the study of the interactions between immune system and tumor microenvironment (TME) in

(c) The Author(s). 2019 Open Access This article is distributed under the terms of the Creative Commons Attribution 4.0 International License (http://creativecommons.org/licenses/by/4.0/), which permits unrestricted use, distribution, and 
brain metastases allowing to identify CNS as an immunologically distinct rather than an immune-isolated compartment [8]. The inflammatory TME of brain metastases has shown to be active in the majority of patients with dense infiltration of tumor-infiltrating lymphocytes (TIL) often expressing immunosuppressive factors like programmed death-1 (PD-1) ligand (PD-L1) [9]. These notions and the recent availability of effective immunotherapeutic agents $[10,11]$, including anticytotoxic T lymphocyte-associated antigen-4 (CTLA-4), anti- PD-1, and PD-L1 monoclonal antibodies (mAbs), have supported their use, also in patients with brain metastases, as well as in primary CNS tumors [12].

In this manuscript we focus on the upcoming clinical evidence demonstrating the effectiveness of immunotherapy with ICI in brain metastases, and on the daily practice implications of these finding. Lastly, we highlight potential future avenues for a more effective immunotherapeutic approach for the treatment of brain metastases.

\section{Brain tumor microenvironment immunobiology}

The tumor microenvironment (TME) of metastatic CNS malignancies, with its highly complex cancer-promoting features, is considered among the main regulators of the response and resistance to treatment [13]. Other than endothelial cells, the brain TME consists of different cell types including fibroblasts, pericytes, microglia and astrocytes, along with a variety of immune cells with suppressive or stimulatory functions [14] physically protected by the blood-brain barrier (BBB). It was shown that the BBB in brain metastases is often compromised, not fully disrupted but rather remodeled into a bloodtumor barrier due to alterations in the pericyte subpopulation [15] encouraging a robust infiltration of multiple immune suppressive cell types from the peripheral circulation [8]. The dynamic interactions occurring between these diverse cell types and cancer cells may contribute to the metastatic progression and may impair response to therapy. Cancer cells metastatic to the brain and astrocytes can stimulate each other directly [16] or through the release of different cytokines and inflammatory mediators, contributing to brain colonization [8]. Indeed, interleukin (IL) -8, macrophage migration inhibitory factor (MIF), and plasminogen activator inhibitor-1 (PAI-1) released by metastatic lung cancer cells were found to be able to activate astrocytes that produced growth factors [i.e., IL-6, IL-1 $\beta$, and tumor necrosis factor- $\alpha$ (TNF- $\alpha)$, thus fostering cancer cell growth in the brain niche [17]. Moreover, in vitro studies demonstrated that neurotrophic factors secreted by reactive astrocytes such as IL-6, transforming growth factor- $\beta$ (TGF- $\beta$ ), insulin-like growth factor-1 (IGF-1), and chemokine ligand 12a (CXCL12a) may contribute to the development of brain metastasis from breast cancer $[18,19]$. In addition, brain-metastasizing melanoma cells were found to reprogram astrocytes to express the proinflammatory cytokine IL-23, which stimulated the secretion of matrix metalloproteinase-2 (MMP-2) enhancing the degradation of the extracellular matrix, and facilitating the extravasation and eventually brain invasion by tumor cells [20].

Besides "resident" astrocytes, type 2 tumor-associated macrophages [21], myeloid-derived suppressor cells (MDSC), regulatory $\mathrm{T}$ cells (T-reg) [14], and cancerassociated fibroblasts (CAF) with pro-tumorigenic characteristics were found to be recruited in the brain by metastatic melanoma, breast and colon cancer [22, 23]. These different cell types were shown to play a negative role in the anti-tumor immune response by reducing the expression of key molecules involved in T-cell costimulation (e.g., CD80, CD86, CD40) [8], impairing antigen presentation [24], and deregulating the homeostasis of the brain microenvironment [8]. In this highly suppressive TME metastatic landscape, TIL are poorly represented and functionally impaired in brain metastases, compared to primary tumors [25]. Along this line, different studies documented a down-regulated T-cell activity as the result of tumor-induced $\mathrm{T}$ cell exhaustion in brain metastasis; indeed, PD-1 expression was detected on $>60 \%$ of TIL [16], though a correlation with clinical outcome remains to be investigated.

Besides the analyses of the different cell population in the brain metastases TME, controversial results have been reported on the exclusive brain metastases molecular profiles. Although several studies demonstrated a genetically divergence [e.g., higher rates of BRAF mutations, higher tumor mutational burden (TMB), higher PD-L1 expression, private gene mutations] between brain metastases and their primary tumors [26-28], no significant differences were observed in mutation profiles between a case series of breast cancer brain metastases and their primary lesions [29]. These findings suggested that additional studies are required to fully identify the unique molecular characteristics/features of brain metastases.

The immunosuppressive role of TME of CNS metastases highlights the need for new therapeutic approaches promoting M1 properties of macrophages, the recruitment of tumor infiltrating CD8 + T cell [30], or targeting suppressive cell types such as T-reg and MDSC. Along this line, it has been demonstrated that the coadministration of a Treg-depleting anti-CD25 mAb and IL-21engineered cell vaccine led to the cure of most mice bearing TS/A micrometastases [31]. Moreover, strategies aiming at converting the immune-suppressive milieus into inflamed environments [31, 32] through the use of antibodies against suppressive cytokines such as TGF- $\beta$, or IL-10 may play a relevant role [33]. In this 
context, the overexpression of TGF- $\beta 2$ by melanoma cells was associated with site-specific brain metastasis; and, accordingly, the use of anti-TGF- $\beta 2$ therapies in a syngeneic murine melanoma model significantly reduced metastasis to the brain [34].

Upcoming findings suggest that epigenetic modeling may also contribute to the immune-suppressive tumor and TME profile of brain metastases. Initial evidence supporting this hypothesis derived from the exploration of the methylomes of lung, breast, and cutaneous melanoma brain metastases, and from their relative primary tumors. These studies allowed building epigenetic classifiers able to determine the origin of brain metastases, the histotype of primary CNS tumors, and also the therapeutic subtype for breast cancer patients [34]. Consistent with the notion that epigenetic modeling plays a relevant role in shaping brain metastases, genes involved in cell development and differentiation, regulation of gene expression, cell migration, and tumor suppression were found to be unmethylated in the majority of breast cancer patients without brain metastases as compared to those with CNS involvement [35]. These findings, and the well-known immunomodulatory potential of DNA hypomethylating agents on genes involved antigen processing and presentation [36], and on immunosuppressive cellular and soluble components of the TME of brain metastases (e.g., MDSC, Treg, chemokines) [37, 38], strongly support the use of epigenetic drugs combined with ICI to develop new strategies for the personalized therapeutic management of patients with brain metastases.

\section{ICI therapy of brain metastases Melanoma}

The initial clinical evidence of ICI activity in melanoma brain metastases was generated in two prospective phase II studies. The first trial investigated the efficacy of ipilimumab in patients with asymptomatic $(n=51$, cohort A) or symptomatic ( $n=21$, cohort B) brain metastases [39]. CNS disease control rate (DCR) at 12 weeks was 24 and $10 \%$, and the intracranial overall response rate (ORR) was 16 and 5\%, in cohorts $A$ and $B$, respectively; median overall survival (OS) was 7 months (range 0.4-31+) for cohort A, and 4 months $(0.5-25+)$ for cohort B, while survival rates at 24 months were 26 , and $10 \%$, respectively. Though initial, these results suggested a better efficacy of treatment in patients with asymptomatic brain metastases and who did not receive steroids. In the phase II Italian Network for Tumor Biotherapy (NIBIT)M1 study, 86 patients with metastatic melanoma received ipilimumab at $10 \mathrm{mg} / \mathrm{kg}$ combined with fotemustine: among the 20 patients who also had asymptomatic brain metastases at study enrolment the ir-DCR was $50 \%$, and it was $46.5 \%$ in the whole population [40].
Also, the 3 year OS was $27.8 \%$ in patients with brain metastases and $28.5 \%$ in the whole population, suggesting a durable clinical benefit of treatment also in patients with asymptomatic brain metastases [41]. A more recent follow-up of this study has shown that 5 complete regressions of brain disease were obtained, with a duration of brain complete response (CR) of $16,28,39,80+, 94+$ months; of note, the 2 patients still alive, in the absence of subsequent treatment, had achieved a CR both intraand extra-cranial (A.M. Di Giacomo, et al. unpublished). Based on this intriguing clinical evidence and on available results showing an additive therapeutic efficacy of ipilimumab combined with nivolumab in melanoma [42], the multicentre, phase III, randomized, open-label NIBIT-M2 study, sponsored by the NIBIT Foundation, was activated [43]. This three-arm study was designed to assess the OS of previously untreated metastatic melanoma patients with asymptomatic brain metastases who received fotemustine, its combination with ipilimumab, or the combination of ipilimumab and nivolumab. Results from a pre-planned interim analysis of the study will be soon available.

Providing additional support to the notion that patients with brain metastases can benefit from ICI treatment, the activity of anti-PD-1 monotherapy was recently reported in a retrospective analysis of 66 melanoma patients with CNS disease treated with nivolumab or pembrolizumab [44]. An intracranial ORR and DCR of 21 and 56\%, respectively, with a median OS of 9.9 months was observed [44]. Moreover, in a prospective phase II study, pembrolizumab induced in 23 melanoma patients an intracranial ORR of $26 \%$, with 2 partial responses (PR) and $4 \mathrm{CR}$. With a median follow-up of 24 months the median progression free survival (PFS) and OS were 2 and 17 months, respectively, and 11 patients (48\%) were still alive at 2 years [45].

Two additional studies have recently investigated the dual blockade of CTLA-4 and PD-1 molecules in melanoma patients metastatic to the brain. The phase II, single-arm, CheckMate 204 study enrolled patients with asymptomatic brain metastases measuring $0.5-3.0 \mathrm{~cm}$, that were treated with a combination of ipilimumab and nivolumab for 4 cycles, followed by nivolumab maintenance until progression or unacceptable toxicity [46]. Among the 94 enrolled patients the intracranial and extracranial ORR were 55 and $50 \%$, respectively, with a global ORR of $51 \%$, and with $90 \%$ ongoing objective responses at a relatively short median duration of followup of 14 months [46]. A recent update of the study with a median follow-up of 20.6 months, reported an intracranial and extracranial ORR of 54 and $49 \%$, respectively, with a global ORR of 51\%, among the 101 evaluable patients with asymptomatic brain metastases; the 18 months survival rate was $75 \%$ [47]. Noteworthy, results 
from a cohort of 18 patients with symptomatic brain metastases demonstrated an intracranial, extracranial, and global ORR of $22 \%$, with a 6 months survival rate of $66 \%$ at a median follow-up of 5.2 months [47]. Consistent with these results are those from the Australian Brain Collaboration (ABC) study, a phase II, prospective trial enrolling 3 cohorts of patients with asymptomatic or symptomatic brain metastases [48]. Subjects with no prior local brain treatment were randomly assigned to receive nivolumab combined with ipilimumab (Cohort A) or nivolumab alone (Cohort B), whereas symptomatic patients who had failed local brain therapy and/or had leptomeningeal spreading disease received nivolumab alone (Cohort C). The intracranial ORR was 46, 20, and $6 \%$ in Cohorts A, B and C, respectively. Among patients enrolled in Cohort $\mathrm{A}$, those with treatmentnaïve brain disease achieved a 56\% ORR while it was $16 \%$ in BRAF mutant patients pre-treated with BRAF and MEK inhibitors [48]. Corroborating the safety results from CheckMate 204 study, treatment-related grade 3/4 adverse events occurred in 19 patients (54\%) in Cohort A, in one patient (4\%) in Cohort B, and in two patients (13\%) in Cohort $C$, with no unexpected toxicities; these findings supported the safety and tolerability of nivolumab alone or in combination with ipilimumab in melanoma patients with brain metastases [48].

Overall, data from these prospective clinical trials demonstrate safety and efficacy of anti-CTLA-4 plus anti-PD-1 therapy, coupled with important ORR, similar to those reported in extracranial sites. The findings are highly encouraging and strongly support the role of ICI therapy also in patients with brain metastases (Table 1). Importantly, additional therapeutic combinations in melanoma patients with brain metastases are being explored, as summarized in Table 2.

\section{Lung Cancer}

As it had previously occurred for melanoma, patients with non-small cell lung cancer (NSCLC) and active brain metastases were excluded from pivotal clinical trials with ICI, and only a few retrospective analyses have presently investigated the efficacy and safety of ICI therapy in this patient population. In a prospective phase II trial pembrolizumab induced an intracranial ORR in 10 out of 34 (29.4\%) PD-L1 ${ }^{+}$patients, with no objective response in the $5 \mathrm{PD}-\mathrm{L1}^{-}$patients treated. The median OS among all patients was 8.9 months, and $31 \%$ of patients were alive at 2 years [49]. A pooled analysis from the three CheckMate studies 063 (phase II), 017 (phase III), and 057 (phase III), explored the role of nivolumab in NSCLC patients with previously treated or untreated asymptomatic brain metastases [50]. Among evaluable patients with pre-treated brain metastases at the time of overall disease progression (PD) or last tumor assessment, 33\% had no evidence of CNS progression while $52 \%$ had progressive brain disease; median OS was longer in the nivolumab group (8.4 months) as compared to the chemotherapy (docetaxel) group (6.2 months). Supporting the efficacy of ICI in NSCLC patients with brain metastases, the Italian expanded access program (EAP) with nivolumab enrolled 409 patients with asymptomatic or pretreated brain metastases who achieved an ORR of $17 \%$ and a DCR of $40 \%$ [51].

In addition, an exploratory subgroup analysis of the OAK study [52], assessing the safety and efficacy of the anti-PD-L1 atezolizumab in patients with or without a history of asymptomatic, treated brain metastases, has shown an acceptable safety profile with a trend toward an OS benefit of atezolizumab versus docetaxel (16 versus 11.9 months). Interestingly, atezolizumab led to a prolonged time to radiologic identification of new symptomatic brain metastases compared with docetaxel [53].

Table 1 Efficacy of immune checkpoint inhibitors in melanoma brain metastases

\begin{tabular}{|c|c|c|c|c|}
\hline Author & Phase & Agent & No. Patients & Intracranial ORR (\%) \\
\hline Margolin (2012) & $\|$ & Ipi & & \\
\hline Cohort A & & & 51 & 16 \\
\hline Cohort B & & & 21 & 5 \\
\hline Di Giacomo (2012) & $\|$ & Ipi + Fotemustine & 20 & 50 \\
\hline Parakh (2017) & Real-world (retrospective) & Nivo or Pembro & 66 & 21 \\
\hline Kluger (2019) & $\|$ & Pembro & 23 & 26 \\
\hline Long (2017) & $\|$ & & & \\
\hline Cohort A & & Ipi + Nivo & 26 & 46 \\
\hline Cohort B & & Nivo & 25 & 20 \\
\hline Cohort C & & Nivo & 16 & 6 \\
\hline Tawbi (2018) & $\|$ & Ipi + Nivo & 94 & 55 \\
\hline
\end{tabular}

Ipi Ipilimumab, Nivo Nivolumab, Pembro Pembrolizumab, ORR Object Response Rate 
Table 2 Summary of ongoing clinical trials with $\mathrm{ICl}$ in solid tumor with brain metastases ${ }^{\mathrm{a}}$

\begin{tabular}{|c|c|c|c|}
\hline $\begin{array}{l}\text { Clinical trial } \\
\text { identifier }\end{array}$ & Trial Name & Phase & Status \\
\hline NCT03175432 & $\begin{array}{l}\text { Study of Bevacizumab in Combination With Atezolizumab in Patients With Untreated Melanoma Brain } \\
\text { Metastases (BEAT-MBM) }\end{array}$ & $\|$ & Recruiting \\
\hline NCT02460068 & $\begin{array}{l}\text { A Study of Fotemustine (FTM) Vs FTM and Ipilimumab (IPI) or IPI and Nivolumab in Melanoma Brain } \\
\text { Metastasis (NIBIT-M2) }\end{array}$ & III & Recruiting \\
\hline NCT03340129 & Anti-PD 1 Brain Collaboration + Radiotherapy (ABC-X Study) (ABC-X) & $\|$ & $\begin{array}{l}\text { Not yet } \\
\text { recruiting }\end{array}$ \\
\hline NCT03728465 & Evaluation of Safety and Efficacy of Patients With Four and More Symptomatic Brain Metastases of Melanoma & $\|$ & Recruiting \\
\hline NCT02681549 & $\begin{array}{l}\text { Pembrolizumab Plus Bevacizumab for Treatment of Brain Metastases in Metastatic Melanoma or Non-small } \\
\text { Cell Lung Cancer }\end{array}$ & $\|$ & Recruiting \\
\hline NCT02858869 & Pembrolizumab and Stereotactic Radiosurgery for Melanoma or Non-Small Cell Lung Cancer Brain Metastases & I & Recruiting \\
\hline NCT03563729 & Melanoma Metastasized to the Brain and Steroids (MEMBRAINS) & $\|$ & Recruiting \\
\hline NCT03873818 & Low Dose Ipilimumab With Pembrolizumab in Treating Patients With Melanoma That Has Spread to the Brain & $\|$ & Recruiting \\
\hline NCT02130466 & $\begin{array}{l}\text { A Study of the Safety and Efficacy of Pembrolizumab (MK-3475) in Combination With Trametinib and } \\
\text { Dabrafenib in Participants With Advanced Melanoma (MK-3475-022/KEYNOTE-022) (patients with inactive brain } \\
\text { metastases eligible) }\end{array}$ & $|/| \mid$ & Recruiting \\
\hline NCT02696993 & $\begin{array}{l}\text { Nivolumab and Radiation Therapy With or Without Ipilimumab in Treating Patients With Brain Metastases } \\
\text { From Non-small Cell Lung Cancer }\end{array}$ & $|/| \mid$ & Recruiting \\
\hline NCT02978404 & Combining Radiosurgery and Nivolumab in the Treatment of Brain Metastases & $\|$ & Recruiting \\
\hline NCT02886585 & Pembrolizumab In Central Nervous System Metastases & $\|$ & Recruiting \\
\hline NCT03867175 & Immunotherapy With or Without SBRT in Patients With Stage IV Non-small Cell Lung Cancer & III & $\begin{array}{l}\text { Not yet } \\
\text { Recruiting }\end{array}$ \\
\hline NCT03719768 & Avelumab With Radiotherapy in Patients With Leptomeningeal Disease & I & Recruiting \\
\hline NCT03325166 & $\begin{array}{l}\text { Pembrolizumab and Magnetic Resonance Imaging With Ferumoxytol in Treating Patients With Non-small Cell } \\
\text { Lung Cancer and Brain Metastases }\end{array}$ & $\|$ & Recruiting \\
\hline NCT02648477 & $\begin{array}{l}\text { Pembrolizumab and doxorubicin hydrochloride or anti-estrogen therapy in treating patients with triple- } \\
\text { negative or hormone receptor-positive metastatic breast cancer }\end{array}$ & $\|$ & Recruiting \\
\hline NCT03483012 & Atezolizumab + Stereotactic Radiation in Triple-negative Breast Cancer and Brain Metastasis & $\|$ & Recruiting \\
\hline NCT03449238 & Pembrolizumab And Stereotactic Radiosurgery (SRS) Of Selected Brain Metastases In Breast Cancer Patients & $|/| \mid$ & Recruiting \\
\hline NCT03526900 & $\begin{array}{l}\text { Atezolizumab in Combination With Carboplatin Plus Pemetrexed in Chemotherapy-naïve Patients With } \\
\text { Asymptomatic Brain Metastasis (ATEZO-BRAIN) }\end{array}$ & $\|$ & Recruiting \\
\hline
\end{tabular}

${ }^{a}$ as of Sep 4, 2019. Source: clinicaltrials.gov

$A B C$ Australian Brain Collaboration, SRS/SBRT stereotactic radiosurgery

Aiming to expand these initial intriguing observations, supporting the role of immunotherapy also in lung cancer patients with brain disease, several ongoing prospective clinical trials are investigating the efficacy and safety of ICI in NSCLC and small cell lung cancer (SCLC) patients with brain metastases (Table 2). Moreover, initial studies aim to explore the role of new prognostic and predictive biomarkers also in NSCLC with brain metastases $[54,55]$.

\section{Renal cell carcinoma}

The 5 year cumulative incidence of brain metastases in renal cell carcinoma (RCC) ranges from 7 to 13\% [56], and limited data are available on the efficacy of current systemic treatment of brain disease in RCC patients. To date the vast majority of prospective trials in RCC allowed the inclusion of patients with stable brain disease, and none of the pivotal trials with ICI reported the efficacy of the immunotherapy in patients with active brain metastases. Initial signs of clinical activity for ICI therapy in brain metastases from RCC derived from case reports and small observational series. Among the latter, the Italian EAP with nivolumab enrolled 389 patients beyond first-line therapy, of whom 32 (8\%) had asymptomatic brain metastases that did not require radiotherapy or high dose steroids (i.e., $>10 \mathrm{mg}$ of prednisone). The 6 and 12 months survival rates of these patients were 87 and $66.8 \%$, and they were 80.0 and $63.1 \%$ in the overall population; the DCR was 53.1 and $53.0 \%$ in patients with or without brain metastases, respectively. Treatment related adverse events (AE) were similar between patients with CNS metastases and the overall population (31\% vs $32 \%)$; however, grade 3-4 toxicities were more frequent $(12 \%$ vs $7 \%)$ in patients with brain disease [57]. The French phase II study GETUG-AFU 26 NIVOREN also evaluated safety and efficacy of nivolumab in metastatic 
RCC after progression on vascular endothelial growth factor receptor (VEGFR)-directed therapies [58]. Seventy-three patients with asymptomatic brain metastases were treated: Cohort A included 39 patients who had not received any prior brain therapy (i.e., surgery, radiation, steroids) while Cohort B included 34 patients who had received prior local treatment (primarily SRS). The primary endpoint was the intracranial ORR in Cohort A according to modified Response Evaluation Criteria in Solid Tumors (RECIST) criteria, allowing target lesions $\geq 5 \mathrm{~mm}$. Intracranial ORR was $12 \%$ in Cohort A but no objective responses were observed in patients with multiple brain lesions or larger than $1 \mathrm{~cm}$. At a median follow up of 23.6 months median duration of treatment was 4.9 months in Cohort A, with $13 \%$ of patients still on therapy at the time of analysis. Interestingly, the intracranial median PFS was 2.7 months and the 12 months OS rate was 67\% [58]. Additional data derived from the phase 3/4 study CheckMate 920 combining treatment with ipilimumab and nivolumab. The study enrolled 28 RCC patients with non-active brain metastases of whom 21 had non-target lesions at baseline (lesions $<10 \mathrm{~mm}$ or previously irradiated): the ORR was $29 \%$ with no CR and 8 PR; median PFS was 9.0 months and the 6 months survival rate was $89 \%$. The incidence of immune-related (ir) AEs was generally consistent with the global safety profile of the combination [59]. These data, despite the limited case series, suggest for the efficacy and safety of ICI also in brain metastases from RCC, though further investigation in prospective clinical trials is needed to draw more solid conclusions on its efficacy in this subset of patients. In fact, recent evidence supports the notion that responsiveness to immunotherapy in RCC with brain disease seems to be multifactorial and heterogeneous, therefore several factors (e.g., TME components, genetic intratumoral heterogeneity, compartment- or location-specific alterations of signaling pathways) need to be further explored to improve efficacy of ICI treatment in RCC with brain metastases [60]..

Table 2 reports the ongoing clinical trial with ICI in patients with RCC metastatic to the brain.

\section{Neuroradiology and immunotherapy response evaluation} Optimal therapeutic management of cancer patients benefits from reliable diagnostic, prognostic and predictive imaging markers, aiming to identify successful treatment prior to changes in tumor size. In the therapeutic management of brain metastases, neuroradiology [61, 62] provides i) number, location, and size of brain metastases at diagnosis, ii) the differential diagnosis versus a wide range of pathologic conditions including primary tumors, vascular malformations, ischemia, hemorrhage, and seizure [63], iii) evaluation of treatment response, and iv) diagnosis of treatment-related AEs or complications. Magnetic resonance imaging (MRI) replaced computed tomography $(\mathrm{CT})$ as the imaging modality of choice for brain metastases in the 1980s. Magnetic field, gradients, advanced sequences, hardware, and software are greatly expanding, as well as image post-processing, allowing quantitative data extraction and analysis capabilities. Positron emission tomography (PET) scanning also has advanced with the more widespread adoption of amino acid tracers replacing traditional [18F]-fluorodeoxyglucose, with improvements in signal-to-noise ratio and diagnostic sensitivity and specificity.

\section{Diagnosis and differential diagnosis}

Head CT is generally reserved to staging and restaging of asymptomatic patients, as well as, in the emergency setting, to rule out hemorrhage, ischemia, and hydrocephalus. Instead, MRI is undoubtedly the gold standard technique that should be utilized in all patients with malignant disease and with a clinical history suggestive for brain metastases [64]. According to the European Society for Medical Oncology guidelines, head MRI screening for brain metastases in patients with unresectable stage III or IV lung cancer is recommended, even though they are neurologically asymptomatic; additionally, head MRI should be utilized prior to curative surgery, regardless of the preoperative stage $[65,66]$. Conversely, brain imaging should not be carried out routinely in asymptomatic metastatic breast cancer patients [67], and for melanoma patients there is currently no consensus on its frequency during the clinical follow-up [68]. The MRI protocol study needs unenhanced T1weighted, fluid attenuated inversion recovery, T2weighted, T2*-weighted or susceptibility-weighted images which clearly differentiates abnormal from normal signals of the nervous tissue. Gadolinium-enhanced T1-weighted magnetic resonance (MR) images are the mainstay of the neuroradiological evaluation of brain metastases since they are easy to perform, and accurately depict the margins of most intra-axial metastases, as well as leptomeningeal, dural-based, and bone metastases. Furthermore, non-morphological or so-called "functional" or "physiological" or "advanced" MR techniques may be useful to further differentiate brain metastases from other neoplastic and non-neoplastic lesions. Most common advanced MR techniques are diffusion weighted imaging with apparent diffusion coefficient measure, perfusion- and permeability-weighted imaging, and MR spectroscopy; however, none of these techniques alone has been proven to be highly specific [62, 69-71]. Thus, a thoughtful synthesis using a combination of these techniques can usually allow the neuroradiologist to correctly discriminate tissues. 


\section{Response evaluation}

Available therapeutic options including surgery, radiation, chemotherapy, and most recently immunotherapy, may significantly affect the imaging features of both brain metastases and brain parenchima, resulting in a quite complex neuroradiological interpretation of post-treatment findings. Notably, the broader application of cancer immunotherapy to patients with brain disease, makes the complexity of neuroradiologic tumor response evaluation increasingly challenging for the neuroradiologist. Clinical responses occurring after initial disease progression or even after the appearance of new lesions, treatmentinduced inflammation, long-term benefit and tumor regression are in fact frequent features in the course of immunotherapy.

Thus, aiming to standardize the radiological evaluation of brain metastases, the Response Assessment in Neuro-Oncology (RANO) Brain Metastases (RANO$\mathrm{BM}$ ) group proposed novel evaluation criteria focused on the objective measurement of tumor size at gadolinium-enhanced T1-weighted MR images, corticosteroid use, and clinical deterioration [72]. The RANO$\mathrm{BM}$ were subsequently incorporated into the immunotherapy RANO (iRANO) criteria [73], providing recommendations for the interpretation of neuroradiological changes in the course of immunotherapy. Specifically, in the absence of worsening neurologic signs, the iRANO recommend a 3 months confirmation of initial $\mathrm{PD}$, within 6 months from the beginning of treatment. If follow-up neuroradiology confirms disease progression, the date of actual progression should be backdated to the date of first neuroradiogical assessment of PD. Also, the appearance of new lesions 6 months or less from the beginning of immunotherapy does not define PD [73].

Of note, the iRANO criteria are limited to intraaxial brain metastases, as leptomeningeal and skull bone metastases are often more difficult to be objectively measured and followed, and thus still rely on a qualitative evaluation. Therefore, the RANO group had initially proposed a Leptomeningeal Assessment in Neuro-Oncology (LANO) scorecard [74] that has recently evolved in a simplified one [75].

The RANO- and iRANO-BM response assessment criteria undoubtedly provide a useful framework for a more effective communication between the neuroradiologist, the neuro-oncologist and clinicians utilizing immunotherapy. Nonetheless, it is imperative for neuroradiologists to be more comprehensively familiar with treatment response criteria and treatment-induced changes of brain lesions [62, 69-71]. Among these are radiation-induced brain injuries that comprise a wide range of neuroradiological findings resulting from fractionated or WBRT [76, 77], and include the development of pseudo-progression of disease that typically occurs within the first 3 months following therapy.

Furthermore, recent evidence suggest that ICI therapy can increase: $i$ ) the rates of hemorrhage of melanoma brain metastases treated with WBRT [78]; ii) the incidence of radiation necrosis after treatment of brain metastases with SRS [79]; iii) tumor pseudo-progressions which typically occur within the first 3 months following therapy and that is thought to represent a milder form of radiation necrosis $[61,80,81]$. Immunotherapy alone can also generate neuroradiological changes that may be misplaced with tumor recurrence or progression [82].

Additionally, despite their prominent role in the RANO and iRANO criteria gadolinium-enhanced T1-weighted images do not breakdown all possible changes occurring after treatment of brain metastases. Thus, non-morphological MR techniques may be useful to further differentiate residual/recurrent tumor from post-treatment changes. A thoughtful synthesis using a combination of these techniques can usually allow the neuroradiologist to correctly discriminate tumor tissues from treatment-induced alterations. Therefore, neuroradiologists need to have a thorough knowledge of available conventional and advanced techniques $[62,69-71,83]$ to evaluate treatment response and potential treatment-related complications. Also, PETbased imaging, especially with aminoacid tracers, provides information on tumor metabolism and is currently under investigation to properly differentiate neoplastic tissues from non-specific, treatment-related changes occurring after surgery, radiotherapy, chemotherapy, and immunotherapy [84-87]. Recommendations on the clinical use of PET in neuro-oncology have been recently reported [84].

\section{Adverse events}

In addition to these multiple challenges in response evaluation of brain metastases, ICI therapy is well acknowledged to lead to ir AEs in a proportion of patients. Among these ir-hypophysitis (HP) can occur during treatment with a higher frequency in patients undergoing CTLA-4 blockade [88]. Notably, the incidence of irHP ranges from 0.5 to $18 \%$, depending on the dose of anti-CTLA-4 therapy utilized, and from its combination with PD-1 blocking agents [89]; conversely, ir-HP is infrequent in patients treated with single agent PD-1/PDL1 blockade. Despite HP must be differentiated from metastatic disease to the hypophysis (accounting for $0.87 \%$ of patients with intracranial metastases), its diagnosis is mainly "presumptive" as no surgery is usually performed. Thus, the diagnosis of HP is mostly based on the association of clinical signs and hormonal deficits and abnormalities, hyponatremia, and/or pituitary imaging abnormalities suggestive for HP. Furthermore, the pituitary gland may appear normal at first MRI, though it does not necessarily rule out HP [90]. 
More rare and potentially fatal ICI-mediated neurologic complications, including limbic encephalitis, aseptic meningitis, Guillain-Barré syndrome, transverse myelitis, myasthenia inflammatory myopathy, and orbital myositis, have been reported [91-93]. These uncommon toxicities and their swift diagnosis and optimal clinical management, undoubtedly necessitate a multidisciplinary team approach that must include the neuroradiologist.

\section{Conclusion}

The forthcoming results of ICI-based therapeutic combination(s) in patients with brain disease may soon lead to significant changes in their comprehensive management, thus revisiting the role of surgery and radiotherapy in CNS metastases. Nevertheless, the efficacy of ICI therapy on brain metastases from tumors where ICI therapy is already the standard of care requires a thoughtful, caseby-case, evaluation on the optimal therapeutic approach to be pursued. In selected cases, ICI therapy alone could indeed represent the optimal therapeutic choice. In this daily practice scenario, as well as when patients with CNS metastases are enrolled in clinical trials, a multidisciplinary interaction is mandatory for their optimal management and must undoubtedly include the neuroradiologist to support treating physicians in evaluating clinical response and neurological side effects.

Upcoming insights from pre-clinical and clinical studies will also allow designing new therapeutic strategies to overcome the limitations deriving from the highly immunosuppressive TME of brain metastases.

\footnotetext{
Abbreviations

ABC: Australian brain collaboration; AE: Adverse event; BBB: Blood-brain barrier; CAF: Cancer-associated fibroblast; CNS: Central nervous system; CR: Complete response; CT: Computed tomography; CTLA-4: Cytotoxic T lymphocyte-associated antigen-4; CXCL12a: Chemokine (C-X-C motif) ligand 12a; DCR: Disease control rate; EAP: Expanded access program: HP: Hypophysitis; ICI (s): Immune checkpoint(s); IGF-1: Insulin-like growth factor-1; IL: Interleukin; IL-10: Interleukin-10; IL-1ß: Interleukin-1ß; IL23: Interleukin-23; IL-6: Interleukin-6; IL-8: Interleukin-8; Ir: Immune-related; iRANO: Immunotherapy RANO; LANO: Leptomeningeal assessment in neuro-oncology; mAb: Monoclonal antibody; MDSC: Myeloid-derived suppressor cell; MIF: Macrophage inhibitory factor; MMP-2: Matrix metallopeptidase 2; MR: Magnetic resonance; MRI: Magnetic resonance imaging; NIBIT: Italian network for tumor biotherapy; NSCLC: No small cell lung cancer; ORR: Overall response rate; OS: Overall survival; PAI1: Plasminogen activator inhibitor-1; PD: Disease progression; PD1: Programmed death-1; PD-L1: Programmed death-ligand 1; PET: Positron emission tomography; PFS: Progression free survival; PR: Partial response; RANO: Response assessment in neuro-oncology; RANO-BM: Response assessment in neuro-oncology brain metastases; RCC: Renal cell carcinoma; RECIST: Response Evaluation Criteria in Solid Tumors; SCLC: Small cell lung cancer; SRS: Stereotactic radiosurgery; TGF- $\beta$ : Transforming growth factor- $\beta$; TIL: Tumor-infiltrating lymphocyte; TMB: Tumor mutational burden; TME: Tumor microenvironment; TNF-a: Tumor necrosis factor-a; Treg: Regulatory t cell; VEGFR: Vascular endothelial growth factor receptor; WBRT: Whole-brain radiation therapy
}

\section{Authors' contributions}

All the authors contributed in the preparation of this work. MM, AMDG, MV and $\mathrm{AC}$ contributed to study conception and design. AMDG, MV, AC, AC, MFL, LC, EG and FP were responsible for collecting the data, analysis and drafting the first copy. AMDG, MV, AC and AC were responsible for editing the manuscript. MM, AMDG, MV and AC were responsible for the final editing and preparation of the manuscript for submission. All authors read and approved the final manuscript.

\section{Funding}

The work was supported in part by grants awarded to Michele Maio from the Associazione Italiana per la Ricerca sul Cancro (AIRC) -ID 153732014 and from the FONDAZIONE AIRC under 5 per Mille 2018 - ID 21073 program.

Availability of data and materials

Not applicable.

Ethics approval and consent to participate

Not applicable.

\section{Consent for publication}

Not applicable.

\section{Competing interests}

Anna Maria Di Giacomo served as on Scientific or Advisory Boards of BristolMyers Squibb, Incyte, Glaxo-Smith Kline, Pierre Fabre;

Monica Valente has not conflict of interest to declare;

Alfonso Cerase has no conflict of interest to declare;

Maria Fortunata Lofiego has not conflict of interest to declare;

Francesca Piazzini has not conflict of interest to declare;

Luana Calabrò served as on Scientific or Advisory Boards of Bristol-Myers

Squibb;

Elisabetta Gambale has not conflict of interest to declare;

Alessia Covre has not conflict of interest to declare;

Michele Maio served as on Scientific or Advisory Boards of Bristol-Myers

Squibb, Roche-Genentech, Astrazeneca-Medlmmune, Merck Sharp \&Dohme and Glaxo-Smith Kline.

\section{Author details}

${ }^{1}$ Department of Oncology, Center for Immuno-Oncology, Medical Oncology and Immunotherapy, University Hospital of Siena, Viale Bracci, 14, 53100 Siena, Italy. ${ }^{2}$ Unit of Neuroradiology, University Hospital, Siena, Italy.

Received: 7 August 2019 Accepted: 23 September 2019

Published online: 17 October 2019

\section{References}

1. Ostrom QT, Wright CH, Barnholtz-Sloan JS. Brain metastases: epidemiology. Handb Clin Neurol. 2018;149:27-42.

2. Arvold ND, Lee EQ, Mehta MP, Margolin K, Alexander BM, Lin NU, et al. Update in the management of brain metastases. Neuro-Oncology. 2016; 18(8):1043-65.

3. Venur VA, Karivedu V, Ahluwalia MS. Systemic therapy for brain metastases. Handb Clin Neurol. 2018;149:137-53.

4. Chamberlain MC, Baik CS, Gadi VK, Bhatia S, Chow LQ. Systemic therapy of brain metastases: non-small cell lung cancer, breast cancer, and melanoma. Neuro-Oncology. 2017;19(1):i1-i24

5. Cagney DN, Martin AM, Catalano PJ, Redig AJ, Lin NU, Lee EQ, et al. Incidence and prognosis of patients with brain metastases at diagnosis of systemic malignancy: a population-based study. Neuro-Oncology. 2017; 19(1):1511-21.

6. Achrol AS, Rennert RC, Anders C, Soffietti R, Ahluwalia MS, Nayak L, et al. Brain metastases. Nat Rev Dis Primers. 2019:5(1):5.

7. Kaufman HL, Atkins MB, Subedi P, Wu J, Chambers J, Mattingly TJ, et al. The promise of Immuno-oncology implications for defining the value of cancer treatment. J Immunother Cancer. 2019;7:129.

8. Quail DF, Joyce JA. The microenvironmental landscape of brain tumors. Cancer Cell. 2017:31(3):326-41.

9. Berghoff AS, Venur VA, Preusser M, Ahluwalia MS. Immune checkpoint inhibitors in brain metastases: from biology to treatment. Am Soc Clin Oncol Educ Book. 2016;35:e116-22. 
10. Rotte A. Combination of CTLA-4 and PD-1 blockers for treatment of cancer. J Exp Clin Cancer Res. 2019;38:255.

11. Tan AC, Heimberger AB, Menzies AM, Pavlakis N, Khasraw M. Immune checkpoint inhibitors for brain metastases. Curr Oncol Rep. 2017;19(6):38.

12. Wang X, Guo G, Guan H, Yu Y, Lu J, Yu J. Challenges and potential of PD-1/ PD-L1 checkpoint blockade immunotherapy for glioblastoma. J Exp Clin Cancer Res. 2019;38(1):87.

13. Juarez TM, Carrillo JA, Achrol AA, Salomon MP, Marzese DM, Park JM, Baliga NS, et al. Understanding the brain tumor microenvironment: considerations to applying systems biology and immunotherapy. Int J Neurooncol. 2018;1:25-33.

14. Quail DF, Joyce JA. Microenvironmental regulation of tumor progression and metastasis. Nat Med. 2013;19(11):1423-37.

15. Lyle LT, Lockman PR, Adkins CE, Mohammad AS, Sechrest E, Hua E, et al. Alterations in pericyte subpopulations are associated with elevated blood tumor barrier permeability in experimental brain metastasis of breast cancer. Clin Cancer Res. 2016;22:5287-99.

16. Farber SH, Tsvankin V, Narloch JL, Kim GJ, Salama AK, Vlahovic G, et al. Embracing rejection: immunologic trends in brain metastasis. Oncoimmunology. 2016;5(7):e11721531-11.

17. Seike T, Fujita K, Yamakawa Y, Kido MA, Takiguchi S, Teramoto N, et al Interaction between lung cancer cells and astrocytes via specific inflammatory cytokines in the microenvironment of brain metastasis. Clin Exp Metastasis. 2011;28(1):13-25.

18. Sierra A, Price JE, García-Ramirez M, Méndez O, López L, Fabra A. Astrocytederived cytokines contribute to the metastatic brain specificity of breast cancer cells. Lab Investig. 1997;77(4):357-68.

19. Hoelzinger DB, Demuth $T$, Berens ME. Autocrine factors that sustain glioma invasion and paracrine biology in the brain microenvironment. J Natl Cancer Inst. 2007:99:1583-93.

20. Wenting $\mathrm{N}$, Wenxing $\mathrm{C}$, Yin L. Emerging findings into molecular mechanism of brain metastasis. Cancer Med. 2018;7(8):3820-33.

21. Hambardzumyan D, Gutmann DH, Kettenmann $\mathrm{H}$. The role of microglia and macrophages in glioma maintenance and progression. Nat Neurosci. 2016;19(1):20-7.

22. Jacobs JF, Idema AJ, Bol KF, Nierkens S, Grauer OM, Wesseling P, et al. Regulatory T cells and the PD-L1/PD-1 pathway mediate immune suppression in malignant human brain tumors. NeuroOncol. 2009;11(4):394-402.

23. Du Four S, Maenhout SK, De Pierre K, Renmans D, Niclou SP, Thielemans K, et al. Axitinib increases the infiltration of immune cells and reduces the suppressive capacity of monocytic MDSCs in an intracranial mouse melanoma model. Oncoimmunology. 2015;22(4):e9981071-17.

24. Liu Y, Komohara Y, Domenick N, Ohno M, Ikeura M, Hamilton RL, et al. Expression of antigen processing and presenting molecules in brain metastasis of breast cancer. Cancer Immunol Immunother. 2012;61(6):789-801.

25. Ogiya R, Niikura N, Kumaki N, Yasojima H, Iwasa T, Kanbayashi C, et al. Comparison of immune microenvironments between primary tumors and brain metastases in patients with breast cancer. Oncotarget. 2017;8(61):103671-81.

26. In GK, Poorman KA, Saul M, O'Day S, Farma JM, Daveluy S. Molecular profiling of melanoma brain metastases (MBM) compared to primary cutaneous melanoma (CM). J Clin Oncol. 2019;37(Suppl 15):9565.

27. Brastianos PK, Carter SL, Santagata S, Cahill DP, Taylor-Weiner A, Jones RT. Genomic characterization of brain metastases reveals branched evolution and potential therapeutic targets. Cancer Discov. 2015;5:1164-77.

28. Saunus JM, Quinn MC, Patch AM, Pearson JV, Bailey PJ, Nones $K$, et al. Integrated genomic and transcriptomic analysis of human brain metastases identifies alterations of potential clinical significance. J Pathol. 2015;237:363-78.

29. Lee JY, Park K, Lim SH, Kim HS, Yoo KH, Jung KS, et al. Mutational profiling of brain metastasis from breast cancer: matched pair analysis of targeted sequencing between brain metastasis and primary breast cancer. Oncotarget. 2015;6:43731-42.

30. Van Woensel M, Mathivet $T$, Wauthoz N, Rosière R, Garg AD, Agostinis $P$, et al. Sensitization of glioblastoma tumor micro-environment to chemo- and immunotherapy by Galectin-1 intranasal knock-down strategy. Sci Rep. 2017;7:1-14.

31. Di Modugno F, Colosi C, Trono P, Antonacci G, Ruocco G, Nisticò P. 3D models in the new era of immune oncology: focus on T cells, CAF and ECM. J Exp Clin Cancer Res. 2019;38(1):117.

32. Looi CK, Chung FF, Leong CO, Wong SF, Rosli R, Mai CW. Therapeutic challenges and current immunomodulatory strategies in targeting the immunosuppressive pancreatic tumor microenvironment. J Exp Clin Cancer Res. 2019;38(1):162.

33. Sevenich L. Turning "cold" into "hot" tumors—opportunities and challenges for radio-immunotherapy against primary and metastatic brain. Front Oncol. 2019;9(163):1-16.

34. Orozco IJ, Manughian-Peter AO, Salomon MP, Marzese DM. Epigenetic classifiers for precision diagnosis of brain tumors. Epigenet Insights. 2019;12:1-3.

35. Shinawi T, Morris M, Pangeni R, Krex D, Schackert G, Latif F. Epigenetic targets in breast cancer brain metastasis. Liverpool: Conference paper at NCRI Cancer Conference; 2014.

36. Coral S, Parisi G, Nicolay HJMG, Colizzi F, Danielli R, Fratta E, et al. Immunomodulatory activity of SGI-110, a 5-aza-2'-deoxycytidine containing demethylating dinucleotide. Cancer Immunol Immunother. 2013;62(3):605-14.

37. Lindau D, Gielen $P$, Kroesen $M$, Wesseling $P$, Adema GJ. The immunosuppressive tumour network: myeloid-derived suppressor cells, regulatory T cells and natural killer T cells. Immunology. 2013;138(2):105-15.

38. Peng D, Kryczek I, Nagarsheth N, Zhao L, Wei S, Wang W, et al. Epigenetic silencing of TH1-type chemokines shapes tumour immunity and immunotherapy. Nature. 2015;527(7577):249-53.

39. Margolin K, Ernstoff MS, Hamid O, Lawrence D, McDermott D, Puzanov I, et al. Ipilimumab in patients with melanoma and brain metastases: an open-label, phase 2 trial. Lancet Oncol. 2012;13(5):459-65.

40. Di Giacomo AM, Ascierto PA, Pilla L, Santinami M, Ferrucci PF, Giannarelli $D$, et al. Ipilimumab and fotemustine in patients with advanced melanoma (NIBIT-M1): an open-label, single-arm phase 2 trial. Lancet Oncol. 2012;13(9):879-86.

41. Di Giacomo AM, Ascierto PA, Queirolo P, Pilla L, Ridolfi F, Santinami M, et al. Three-year follow-up of advanced melanoma patients who received ipilimumab plus fotemustine in the Italian network for tumor biotherapy (NIBIT)-M1 phase II study. Ann Oncol. 2015;26(4):798-803.

42. Hodi FS, Chiarion-Sileni V, Gonzalez R, Grob JJ, Rutkowski P, Cowey CL, et al. Nivolumab plus ipilimumab or nivolumab alone versus ipilimumab alone in advanced melanoma (CheckMate 067): 4-year outcomes of a multicentre, randomised, phase 3 trial. Lancet Oncol. 2018;19(11):1480-92.

43. Di Giacomo AM, Annesi D, Ascierto PA, Calabro L, Chiarion-Sileni V, Danielli $R$, et al. A randomized, phase III study of fotemustine versus the combination of fotemustine and ipilimumab or the combination of ipilimumab and nivolumab in patients with metastatic melanoma with brain metastasis: the NIBIT-M2 trial. J Clin Oncol. 2015;33(Suppl 15):abs 9090.

44. Parakh S, Park JJ, Mendis S, Rai R, Xu W, Lo S, et al. Efficacy of anti-PD-1 therapy in patients with melanoma brain metastases. Br J Cancer. 2017; 116(12):1558-63.

45. Kluger HM, Chiang V, Mahajan A, Zito CR, Sznol M, Tran T, et al. Long-term survival of patients with melanoma with active brain metastases treated with pembrolizumab on a phase II trial. J Clin Oncol. 2019;37(1):52-60.

46. Tawbi HA, Forsyth PA, Algazi A, Hamid O, Hodi FS, Moschos SJ, et al. Combined Nivolumab and Ipilimumab in melanoma metastatic to the brain. N Engl J Med. 2018;379(8):722-30.

47. Tawbi HA, Forsyth PA, Hodi FS, Lao CD, Moschos SJ, Omid A, et al. Efficay and safety of the combination of nivolumab (NIVO) plus ipilimumab (IPI) in patients with symptomatic melanoma brain metastases (ChechMate 204). J Clin Oncol. 2019:37:abs 9501.

48. Long GV, Atkinson V, Lo S, Sandhu S, Guminski AD, Brown MP, et al. Combination nivolumab and ipilimumab or nivolumab alone in melanoma brain metastases: a multicentre randomised phase 2 study. Lancet Oncol. 2018;19(5):672-81.

49. Goldberg SB, Gettinger SN, Mahajan A, Herbst RS, Chiang AC, Lilenbaum R, et al. Durability of brain metastasis response and overall survival in patients with non-small cell lung cancer (NSCLC) treated with pembrolizumab. J Clin Oncol. 2018;36(Suppl 15):2009.

50. Goldman JW, Crinò L, Vokes EE, Holgado E, Reckamp KL, Pluzanski A, et al. Nivolumab in patients with advanced NSCLC and central nervous system metastases. J Clin Oncol. 2016;34(Suppl 15):9038.

51. Crinò L, Bronte G, Bidoli P, Cravero P, Minenza E, Cortesi E, et al. Nivolumab and brain metastases in patients with advanced non-squamous non-small cell lung cancer. Lung Cancer. 2019;129:35-40.

52. Rittmeyer A, Barlesi F, Waterkamp D, Park K, Ciardiello F, von Pawel J, et al. Atezolizumab versus docetaxel in patients with previously treated non-small-cell lung cancer (OAK): a phase 3, open-label, multicentre randomised controlled trial. Lancet. 2017;389(10066):255-65. 
53. Gadgeel SM, Lukas RV, Goldschmidt J, Conkling P, Park K, Cortinovis D, et al. Atezolizumab in patients with advanced non-small cell lung cancer and history of asymptomatic, treated brain metastases: exploratory analyses of the phase III OAK study. Lung Cancer. 2019;128:105-12.

54. Takamori S, Takada K, Azuma K, Jogo Y, Kinoshita F, Kozuma Y, et al. Prognostic impact of PD-L2 expression and association with PD-L1 in patients with small-cell lung Cancer. Anticancer Res. 2018;38(10):5903-7.

55. Wang Q, Lin W, Tang X, Li S, Guo L, Lin Y, et al. The roles of microRNAs in regulating the expression of PD-1/PD-L1 immune checkpoint. Int J Mol Sci. 2017:18(12):2540.

56. Kattan J, Rassy EE, Assi T, Bakouny Z, Pavlidis N. A comprehensive review of the role of immune checkpoint inhibitors in brain metastasis of renal cell carcinoma origin. Crit Rev Oncol Hematol. 2018;130:60-9.

57. Bracarda S, Galli L, Maruzzo M, Lo Re G, Buti S, Favaretto A, et al. Negative prognostic factors and resulting clinical outcome in patients with metastatic renal cell carcinoma included in the Italian nivolumab-expanded access program. Future Oncol. 2018;14(14):1347-54.

58. Flippot R, Dalban C, Laguerre B, Borchiellini D, Gravis G, Negrier S, et al. Safety and efficacy of nivolumab in brain metastases from renal cell carcinoma: results of the GETUG-AFU 26 NIVOREN multicenter phase II study. J Clin Oncol. 2019;37(23):2008-16.

59. Emamekhoo H, Olsen M, Carthon B, Drakaki A, Percent I, Molina AM, et al. Safety and efficacy of nivolumab plus ipilimumab in patients with advanced renal cell carcinoma with brain metastases: interim analysis of CheckMate 920. J Clin Oncol. 2019:3715(Suppl):4517.

60. Reed JP, Posadas EM, Figlin RA. Brain metastases in renal cell carcinoma: immunotherapy responsiveness is multifactorial and heterogeneous. J Clin Oncol. 2019;37(23):1987-9.

61. Pope WB. Brain metastases: neuroimaging. Handb Clin Neurol. 2018;149:89-112.

62. Kessler AT, Bhatt AA. Brain tumour post-treatment imaging and treatmentrelated complications. Insights Imaging. 2018;9(6):1057-75.

63. Muccio CF, Tarantino A, Esposito G, Cerase A. Differential diagnosis by unenhanced FLAIR T2-weighted magnetic resonance images between solitary high grade gliomas and cerebral metastases appearing as contrastenhancing cortico-subcortical lesions. J Neuro-Oncol. 2011;103(3):713-7.

64. Soffietti R, Cornu P, Delattre JY, Grant R, Graus F, Grisold W, et al. EFNS guidelines on diagnosis and treatment of brain metastases: report of an EFNS task force. Eur J Neurol. 2006;13(7):674-81.

65. Eberhardt WE, De Ruysscher D, Weder W, Le Péchoux C, De Leyn P, Hoffmann $H$, et al. 2nd ESMO consensus conference in lung Cancer: locally advanced stage III non-small-cell lung cancer. Ann Oncol. 2015;26(8):1573-88.

66. O'Dowd EL, Kumaran M, Anwar S, Palomo B, Baldwin DR. Brain metastases following radical surgical treatment of non-small cell lung cancer: is preoperative brain imaging important? Lung Cancer. 2014;86(2):185-9.

67. Cardoso F, Costa A, Senkus E, Aapro M, André F, Barrios CH, et al. 3rd ESOESMO international consensus guidelines for advanced breast Cancer (ABC 3). Ann Oncol. 2017:28(1):16-33.

68. Dummer R, Hauschild A, Lindenblatt N, Pentheroudakis G, Keilholz U. ESMO Guidelines Committee. Cutaneous melanoma: ESMO Clinical Practice Guidelines for diagnosis, treatment and follow-up. Ann Oncol. 2015; 26(Suppl 5):v126-32.

69. Nowosielski M, Radbruch A. The emerging role of advanced neuroimaging techniques for brain metastases. Chin Clin Oncol. 2015:4(2):23.

70. Villanueva-Meyer JE, Mabray MC, Cha S. Current clinical brain tumor imaging. Neurosurgery. 2017:81(3):397-415.

71. Nowosielski M, Wen PY. Imaging criteria in Neuro-oncology. Semin Neurol. 2018;38(1):24-31.

72. Lin NU, Lee EQ, Aoyama H, Barani IJ, Barboriak DP, Baumert BG, et al. Response assessment in Neuro-oncology (RANO) group. Response assessment criteria for brain metastases: proposal from the RANO group. Lancet Oncol. 2015;16(6):e270-8.

73. Okada H, Weller M, Huang R, Finocchiaro G, Gilbert MR, Wick W, et al. Immunotherapy response assessment in neuro-oncology: a report of the RANO working group. Lancet Oncol. 2015;16(15):e534-42.

74. Chamberlain M, Junck L, Brandsma D, Soffietti R, Rudà R, Raizer J, et al. Leptomeningeal metastases: a RANO proposal for response criteria. NeuroOncology. 2017;19(4):484-92.

75. Le Rhun E, Devos P, Boulanger T, Smits M, Brandsma D, Rudà R, et al. European Organisation for Research and Treatment of Cancer (EORTC) brain tumor group (BTG) central nervous system (CNS) metastases committee and the EORTC BTG imaging committee. The RANO Leptomeningeal metastasis group proposal to assess response to treatment: lack of feasibility and clinical utility and a revised proposal. Neuro-Oncology. 2019;21(5):648-58,

76. Greene-Schloesser D, Robbins ME, Peiffer AM, Shaw EG, Wheeler KT, Chan MD. Radiation-induced brain injury: a review. Front Oncol. 2012;2:73.

77. Valk PE, Dillon WP. Radiation injury of the brain. AJNR Am J Neuroradiol. 1991;12(1):45-62.

78. Gerber NK, Young RJ, Barker CA, Wolchok JD, Chan TA, Yamada Y, et al. Ipilimumab and whole brain radiation therapy for melanoma brain metastases. J Neuro-Oncol. 2015;121:159-65.

79. Colaco RJ, Martin P, Kluger HM, Yu JB. Does immunotherapy increase the rate of radiation necrosis after radiosurgical treatment of brain metastases? Neurosurg. 2016;125:17-23.

80. Cohen JV, Alomari AK, Vortmeyer AO, Jilaveanu LB, Goldberg SB, Mahajan A et al. Melanoma brain metastasis Pseudoprogression after Pembrolizumab treatment. Cancer Immunol Res. 2016:4(3):179-82.

81. Pires da Silva I, Glitza IC, Haydu LE, Johnpulle R, Banks PD, Grass GD, et al. Incidence, features and management of radionecrosis in melanoma patients treated with cerebral radiotherapy and anti-PD-1 antibodies. Pigment Cell Melanoma Res. 2019;32(4):553-63.

82. Galldiks N, Kocher M, Ceccon G, Werner JM, Brunn A, Deckert M, et al. Imaging challenges of immunotherapy and targeted therapy in patients with brain metastases: response, progression, and Pseudoprogression. Neuro-Oncology. 2019. https://doi.org/10.1093/neuonc/noz147.

83. Fabi A, Vidiri A. Defining the endpoints: how to measure the efficacy of drugs that are active against central nervous system metastases. Transl Lung Cancer Res. 2016;5(6):637-46.

84. Albert NL, Weller M, Suchorska B, Galldiks N, Soffietti R, Kim MM, et al. Response assessment in Neuro-oncology working group and European Association for Neuro-Oncology recommendations for the clinical use of PET imaging in gliomas. Neuro-Oncology. 2016;18(09):1199-208.

85. Kebir S, Rauschenbach L, Galldiks N, Schlaak M, Hattingen E, Landsberg J, et al. Dynamic O-(2-[18F]fluoroethyl)-L-tyrosine PET imaging for the detection of checkpoint inhibitor-related pseudoprogression in melanoma brain metastases. Neuro-Oncology. 2016;18(10):1462-4.

86. Langen KJ, Galldiks N, Hattingen E, Shah NJ. Advances in neuro-oncology imaging. Nat Rev Neurol. 2017;13(05):279-89.

87. Dimitrakopoulou-Strauss A. Monitoring of patients with metastatic melanoma treated with immune checkpoint inhibitors using PET-CT. Cancer Immunol Immunother. 2019;68(5):813-22.

88. Solinas C, Porcu M, De Silva P, Musi M, Aspesiagh S, Scartozzi M, et al. Cancer immunotherapy-associated hypophysitis. Semin Oncol. 2018;45(3):181-6.

89. de Filette J. A Systematic Review and Meta-Analysis of Endocrine-Related Adverse Events Associated with Immune Checkpoint Inhibitors. Horm Metab Res. 2019:51(03):145-56.

90. Briet C, Albarel F, Kuhn E, Merlen E, Chanson P, Cortet C. Expert opinion on pituitary complications in immunotherapy. Ann Endocrinol (Paris). 2018; 79(5):562-8.

91. Bertrand A, Kostine M, Barnetche T, Truchetet ME, Schaeverbeke T. Immunerelated adverse events associated with anti-CTLA-4 antibodies: systematic review and meta-analysis. BMC Med. 2015;13:211.

92. Fay AP, Brandão Moreira R, Nunes Filho RSP, Albuquerque C, Barrios CH. The management of immune-related adverse events associated with immune checkpoint blockade. Expert Rev Qual Life Cancer Care. 2016;1:189-97.

93. Savoia P, Astrua C, Fava P. Ipilimumab (anti-Ctla-4 Mab) in the treatment of metastatic melanoma: effectiveness and toxicity management. Hum Vaccin Immunother. 2016;12(5):1092-101.

\section{Publisher's Note}

Springer Nature remains neutral with regard to jurisdictional claims in published maps and institutional affiliations. 